\title{
27. COMMISSION DES ETOILES VARIABLES
}

PrÉsident: M. H. Shapley, Director of the Harvard College Observatory, Cambridge, Mass., U.S.A.

Vice-Président: M. A. A. Nijland, Director of the Observatory, Utrecht, Holland.

Membres: MM. Ascarza, Baldwin, Banachiewicz, Bemporad, Mlle Blagg, MM. Blažko, A. N. Brown, L. Campbell, Mlle Cannon, MM. Cox, Danjon, De Roy, Dugan, Gerasimovič, Graff, Grouiller, Guthnick, Henroteau, Hertzsprung, Hoffmeister, Jordan, Kordylewski, Kristensen, Lacchini, Ludendorff, LuplauJanssen, McLaughlin, Mascart, Merrill, D. B. Pickering, Prager, Ryves, Schilt, Shinjo, Silva, Stebbins, Stein, Sternberk, E. Strömgren, ten Bruggencate.

Fourteen members of the committee have failed to respond to correspondence; nearly all of the others have expressed the definite opinion that non-responsive members should be dropped from committee membership when a new list is prepared, a few correspondents indicating, however, that the policy should be adopted for Commission 27 only if generally adopted for all commissions of the Union.

It would obviously be impossible to give a summary even of the most important contributions to variable star astronomy during the past four years. It is of interest to note that about fifteen per cent. of the papers published in the principal astronomical journals and observatory publications deal directly or indirectly with variable stars.

Correspondence with various members of the VariableStar Commission shows that two matters are so much in the minds of the members that they deserve preferential consideration in discussing those problems in the study of variable stars that merit international attention. The two subjects are the establishment of comparison star sequences, and the problem of the reduction and publication of old series of observations.

A. So many observers have emphasized the urgent need of comparison star sequences that the chairman recomniends that the Commission deal specifically with this problem during its sessions at the coming meeting of the Union. It may be advisable to empower a small sub-committee to canvass the needs of observers and to take steps to encourage systematic work on sequences. Several writers urge that sequences for visual work on long period and irregular variable stars be established by standardized photovisual means. Recently Miss Payne at the Harvard Observatory has published photovisual magnitude sequences in the Standard Regions, and an extensive programme has been inaugurated to determine the photovisual magnitudes of the comparison stars for a large number of bright southern variables. In co-operation with the Vatican Observatory she is also determining photovisually the magnitudes of comparison stars in the fields of Series VIII of the Atlas Stellarum Variabilium.

Mr de Roy has pointed out the importance of reducing observations of long period variable stars to the International photovisual scale as soon as a uniform and well-standardized system of photovisual sequences is available. He also urges that steps be taken by visual observers to determine and publish their "colour errors."

B. The publication of long series of variable star observations, now filed in various observatories and private libraries, will be of much importance in studying the changes in the periods of variable stars, and of some value in investigations of 
changes in light curves, colours, and amplitudes of variation. Economic conditions throughout the world make it impossible at present to publish in detail the numerous important old series of observations, but the expense of publishing a summary of the data about unpublished observations should not be great. The chairman suggests, on the recommendation of several members, that the Commission take steps to collect during the next year all available details about unpublished observations of variable stars and prepare a report for publication by the International Astronomical Union.

When such a report is published, giving for each observer the names of the stars observed, the numbers of observations of each star in each year, the location of the published observations, the conditions under which the observations will be lent or transcribed, and similar data, the student of a given star will be able to plan a monographic investigation satisfactorily.

A few further points that may be of interest to the Commission are listed below:

I. The systematic investigation of the Cepheid variable stars in ten fields, taken up experimentally by the Commission after the Leiden meeting of 1928 , has not progressed far enough to merit the proposed systematic discussion of the project. The chairman recommends that a comprehensive examination of the results obtained by the many observers who have taken part in this programme be postponed until more of the results have been completed and published. It will suffice to say now that observations, mostly photographic, have been made at a dozen different observatories on practically all of the fifty Cepheid variables, and that in the preparation of the photographic charts and the determination of photographic magnitude sequences at Ottawa and Harvard some valuable equipment has been placed in the hands of variable star observers. Half a dozen American observatories and departments of astronomy have undertaken the measurement and discussion of the variable star photographs made in the course of this campaign with the 8-inch Draper telescope at the Harvard Observatory. Apart from Dr Robinson's studies of the light curves and periods of the "International" Cepheids on the basis of photographs already existing in the Harvard collection, very little of the accumulating material on individual stars has been completed and printed.

2. Suggestions have been made by Baldwin, de Roy, Olcott, and others concerning the reorganization of the observing lists of the various variable star associations. Perhaps this matter should again be discussed in open meeting when the Commission meets again. De Roy urges a systematic and not prolonged study of the more than two thousand long period variables for which no satisfactory light curves have been determined; those having maxima between 9.5 and II.O might be studied systematically either by visual observers or with the Harvard plates.

3. The publication of identification charts of variable stars and their accompanying sequences is advocated by Strömgren. Especially for the new variables that are bright enough or interesting enough to be studied in places other than the discovery point, charts should be published as promptly as possible.

4. Dr Merrill calls attention to the excellent opportunity for spectrophotometric work on red variable stars. "Ordinary colour indices for these stars should not be stressed, as they are difficult to interpret and may be misleading. In the M-type variables the titanium bands very greatly reduce the apparent intensity of the continuous spectrum over a long range from the blue to the red. It is therefore important to observe intensities in the violet or ultra-violet, the red, and the infra- 
red. Observations near $\lambda \lambda 3900,4080,655^{\circ}$, and 8400 , for example, would be particularly interesting. The last-mentioned wave-length can now be recorded on neocyanine plates, while the next to the last is easily observed on several types of commercial panchromatic plates."

5. Dr Schilt recommends that variable star workers note the importance of more accurate determinations of the periods of cluster type variables, especially in globular clusters that have large numbers of variable stars. Without more extensive observations and more accurately determined periods important problems of the variations in periods cannot be satisfactorily attacked. Dr Schilt raises the question whether or not the Commission should recommend co-operation in this field. The chairman would like to suggest that series of plates with large reflectors could be made for measurement and discussion at other observatories.

HaRLow Shapley

President of the Commission

\section{Appendix}

As an appendix to this report, excerpts are given from the correspondence of four members of the Commission.

\section{COMPARISON STARS, COLOUR EQUATIONS, AND PERIODS OF ECLIPSING BINARIES}

\section{By Raymond S. Dugan}

The problem of getting full value from the large number of intrinsically good estimates of variables of short period, especially eclipsing variables, must be given more study. The transformation from step scale to magnitude scale cannot be made directly with photometric magnitudes of the comparison stars for, due to personal equation in colour, distance, and position angle, the observer may see certain comparison stars much brighter or fainter than they are measured photometrically. The essential thing is to discover how bright the observer sees them. Due to the scarcity of nearby bright comparison stars, the difficulties of measuring the brightness of the faint comparison stars, and the usual lack of linearity of the relation between steps and magnitudes at low brightness, the total range of variation, which should be determined with the greatest care, is usually quite uncertain. The regular use of several comparison stars fainter than the variable at minimum will help a great deal. Several comparison stars brighter than the variable at maximum are equally desirable but usually not to be found close enough to the variable. Magnitudes of faint comparison stars estimated by limiting apertures are of doubtful value. More impersonal magnitudes of comparison stars can probably be obtained by measuring photovisual images with an instrument such as the Schilt photometer than by visual photometric observation.

There is a great need for the colour indices of a large number of eclipsing variables at maximum and minimum, in order to determine the types of the components. They have been derived for a few stars from a comparison of visual and photographic curves. This is a slow process and may be unsatisfactory owing particularly to the possible effect of colour on the visual curve. The colour indices should be found by comparison of photovisual and photographic observations. A few observations of each star at appropriate times will suffice. Spectral types at maximum

S A. U IV 
and minimum also should be determined. Even where there is a considerable difference in surface brightness of the two components the spectra are often reported as very similar.

Colour indices or spectral types of the variable and the comparison stars are needed also in any allowance for a possible distortion of the observed light curve due to colour sensitivity. This may well be very important and necessitate a revision of some published light curves. It is not always possible to select comparison stars of the same spectral type as the variable at maximum and even when this can be done there still remains the change of spectral type of the variable as it goes into eclipse. Proper allowance also cannot be made until the observer has carried through a thorough test to determine how the colour sensitivity of his eye changes with brightness. A test under customary observing conditions is probably to be preferred to a laboratory test. For such a test with a polarizing photometer one would need a quite small standard field containing stars of a considerable range in brightness of well-determined colour indices. The North Polar Sequence is too scattered for this purpose and the manipulation of an equatorial at that declination is awkward.

The problem of the variation of period of eclipsing variables is still largely unsolved. The complexity of the problem and its bearing on the density gradient within a star have been emphasized by recent studies on Y Cygni. It is important that observation of this star should be continued. The period introduces interruptions of several years in the observation of minima at any one place and international co-operation in this case is peculiarly appropriate. Other stars with comparably deep minima and eccentricity of orbit and which promise to show a fairly uncomplicated revolution of the line of apsides are RU Monocerotis and YY Sagittarii. There are a number of other stars with varying period.

A few hours' work each year by one observer will provide homogeneous material on the times of minima of one star. RT Persei has been followed in this way for twenty-six years. U Cephei is also regularly observed. A more frequent determination of the time of minimum of a suitable star may uncover the rapid revolution of the line of apsides which may be expected. The large eccentricity of U Cephei, determined spectrographically, should be checked by further observation.

Writers should be careful to state whether the times given are heliocentric.

\section{REDUCTION OF OBSERVATIONS OF LONG PERIOD VARIABLES TO THE INTERNATIONAL PHOTOVISUAL SCALE}

\section{By FELIX DE RoY}

The question may be raised whether the time has not come to devise means of putting the construction of visual light curves of deep yellow and reddish Variable Stars with periods of over I0o $^{\mathrm{d}}$ on a more rational basis.

These curves are now obtained in most cases by collecting into simple arithmetical means the observations made by various individual observers over certain periods of time ( 5 or Io days). Accidental errors are thus eliminated, and systematic errors reduced to a certain extent, but the process cannot lay claim to even moderate accuracy.

The problem of perfecting the curves through elimination of systematic errors can be seemingly approached in two ways.

The first consists in asking the various observers to determine and to publish 
their colour error, i.e. roughly, the quantity whereby they see a red star brighter or fainter than its value on the photovisual scale, when comparing it with white or whitish stars.

H. Grouiller (Bull. Obs. Lyon, 8, 7 c, May I926) has suggested that observers list the stars of the Polar Sequence in the order in which they see them, and estimate the difference between these stars in steps. J. Cox (C.R. Congrès Nat. Sci., Brussels, I931, p. 482) has devised a method by which observers compare selected red stars of the Polar Sequence, and this list has been partly observed by Am. Dermul. The French Association of Variable Stars Observers has published excellent Charts of the Polar Sequence well adapted to this procedure. In I93I, I have made a larger series of similar comparisons $(89)$, extending from $5^{\mathrm{m} \cdot 2}$ to $\mathrm{I} 3^{\mathrm{m}} \cdot 8$. Their discussion shows that, in accordance with the findings of Seares, my colour correction is a function of both magnitude and Colour Index of the stars observed. If the simple formula which represents this correction were known for every observer, and the correction applied to deduced magnitudes, less scattering of the individual observations and improved means might be obtained.

A second and closer method consists in the use of coloured screens, or filters, defining comparatively small portions of the spectrum. The Colour Index of the comparison stars being known, they will give directly the magnitude of the Variable in the photovisual system.

$\mathrm{J}$. van der Bilt and myself have made numerous observations of $U$ Cygni and $R$ Leonis in this way, several right through minimum, using a 4 -inch refractor and an 8 -inch reflector, in order to test the method, and to gather data on the best filter to be used, which should represent a compromise between monochromatism and absorption. Six filters, glass and gelatine, by various makers, were used for most of the observations. Their results have been discussed at Utrecht with the aid of $M$. Minnaert, of the Heliophysical Institute, who determined the absorption curves of the filters, and made various tests on the colour sense of the two observers. They have been published in the March I932 number of the M.N.R.A.S.

This method postulates the knowledge of the spectra, or at least the colour indices of the comparison stars. The latter may be obtained conveniently by measuring with the Schilt photometer plates made in blue and yellow light, alternately on the field of the Variable and on the Polar Sequence. Tests to this effect are being made by $\mathrm{P}$. Bourgeois, using the I2-inch Uccle astrograph, and the plates will be measured by the courtesy of A. Pannekoek at the Astronomical Institute, Amsterdam. If this procedure could be extended to a large number of fields, it would supply, at least down to $12^{\mathrm{m}}$, these "fundamental" comparison stars proposed by P. M. Ryves (Trans. I.A.U. 3, I59), which are greatly needed, existing sequences of comparison stars for long periods, even the best ones, being far from forming a homogeneous system, quite apart from their accidental errors.

\section{THE NEED FOR COMPARISON STAR MAGNITUDES}

\section{By A. A. Nijland}

Personally I would lay stress on a weak point of visual variable star work: the lack of trustworthy magnitudes of comparison stars.... Comparison stars are chiefly wanted for the old well-known long period variable stars of which many thousands of observations lie waiting for reduction, which cannot satisfactorily be carried out without a sound basis of photometric magnitudes. Of course Harvard 
Annals 63 gives the comparison stars for several hundreds of stars; but since the appearance of this volume very few lists of comparison stars have been published, so far as I am aware. And trustworthy magnitudes of comparison stars for many other long period variables are very much wanted. I would suggest photovisual magnitudes, determined for series of comparison stars for these variables, and also for, say, twenty or thirty of the old ones already given in Harvard Annals 37, 57, and 63, in order to be able to compare the photovisual with the visual photometric scale.

The long period variable stars for which photometric magnitudes of comparison stars are wanted are listed below. As a rule the stars with ranges less than two magnitudes have been omitted. Stars in parentheses are less urgent, since their light curves are known less accurately, for the time being. A few stars from List A (B.A.N. 197) have been added.

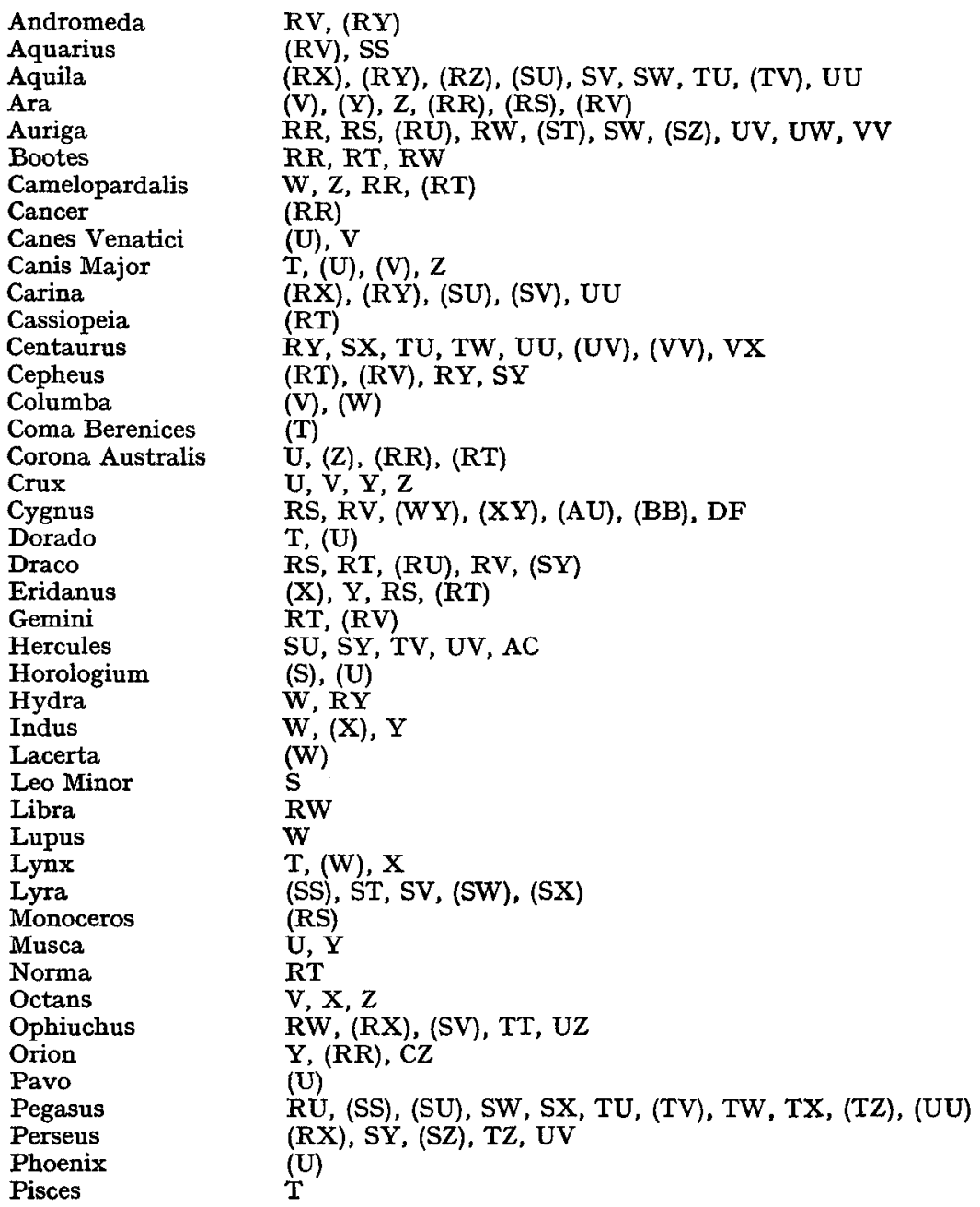




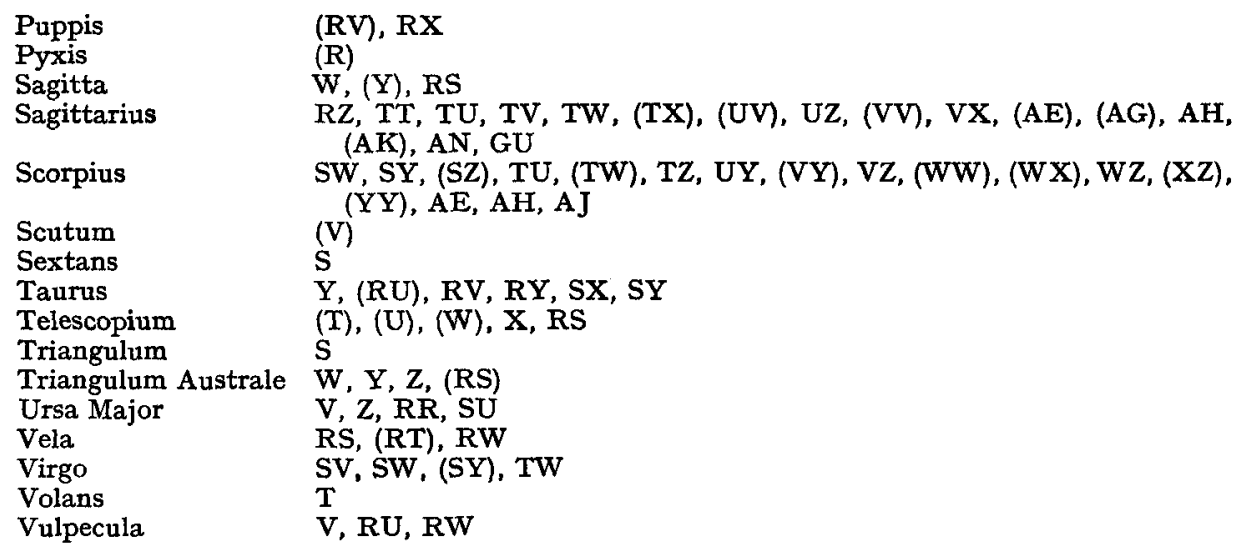

\section{A SUGGESTION FOR INCREASING THE EFFICIENCY OF VARIABLE STAR OBSERVING \\ By William Tyler Olcott}

The members of the A.A.V.S.O. are now observing in the neighbourhood of $45^{\circ}$ long period Variable Stars annually.

It seems to me that a discussion of the curves, and the classification already established, would indicate that a large number of Variables fall into groups or types as indicated by peculiarities in their curves.

The necessity for observing continuously each member of a specific group save in the case of the irregular Variables involves a waste of time.

Why not take four or eight stars in each group from the seasonal constellations so as to have these types continuously observed, concentrate on these, and drop the others from the observing list?

Instead of having sporadic observations for all the stars we would have a large number for each example of a group or type.

In the case of some groups it might not be wise to do this, owing to irregularities in the curve or variations in the duration of maximum and minimum, but there must be a large group where we find a smooth sine curve repeated continuously.

I take it that it is desirable to study the types of variability. If this is the case a few of each group would be sufficient rather than observations of all stars of a class, especially when some classes must embrace fifty or more stars. 